\title{
Utilisation digestive de rations riches en lignines chez le lapin en croissance : mesures de flux et de transit dans différents segments digestifs
}

\author{
T. GIDENNE \\ avec la collaboration technique de A. LAPANOUSE \\ et Véronique TARTIE \\ INRA, Laboratoire de Recherche sur l'Elevage du Lapin, \\ Centre de Recherches de Toulouse \\ B.P. 27, F 31326 Castanet Tolosan Cedex
}

\section{Résumé}

La dégradation des constituants pariétaux ainsi que leur rôle à l'égard de la digestion de la ration sont mal connus chez le lapin. L'effet de hautes teneurs en lignines sur la digestion, les flux et le transit digestif a été mesuré sur trois lots de 36 lapereaux recevant ad libitum un aliment riche en fibres (50 p. 100 de NDF, à base de luzerne déshydratée et de pellicules de colza), caractérisé essentiellement par sa teneur variable en lignines VAN SoEST : 7,4 (lot B) ; 12,8 (lot M) et 16,0 p. 100 MS (lot $\mathrm{H}$ ). Les mesures de flux et de transit utilisent une technique d'abattages, répartis sur 24 heures, après ingestion d'aliment marqué au chrome mordancé.

L'élévation de la teneur en lignines alimentaire induit une diminution modérée de l'efficacité digestive : la digestibilité des protéines est plus particulièrement affectée (CUDa $=66,1 \%$, lot $\mathrm{B} v s$ $60,5 \%$, lot $\mathrm{H}$ ), tandis que les CUDa de l'énergie et de la cellulose ne sont pas significativement modifiés. Les lignines, réputées indigestibles, présentent un CUDa de 14,0 (lot $\mathrm{H}$ ) à 23,0 p. 100 (lot B), ce qui pose le problème de la validité de la technique de VAN SoEST appliquée à l'analyse de produits particuliers tels que les pellicules de colza ou les fèces.

Les bilans digestifs iléaux font apparaitre une digestibilité élevée des hémicelluloses et une digestibilité négative de l'azote. Par contre, la digestibilité mesurée par collecte rectale est identique à celle obtenue par récolte totale des fèces : en effet à ce niveau, les digesta (sous forme de crottes) ne présentent pas de vitesses de transit différentielles et permettent un échantillonnage correct. La représentativité des digesta (excepté dans le rectum) obtenus par abattages est donc mise en cause. Cependant, notre principe des mesures de flux chez le lapin, tenant compte de la caecotrophie, est confirmé.

Les temps de rétention apparents (Minson, 1966) des constituants pariétaux (TRa) sont plus élevés dans le caecum $(3,0 \mathrm{~h}$ à $5,0 \mathrm{~h})$ que dans l'estomac $(1,7 \mathrm{à} 4,0 \mathrm{~h})$. Lorsque la teneur en lignines s'élève, cet écart diminue, du fait d'une hausse des TRa dans l'estomac. Le TRa des constituants augmente avec leur indigestibilité, les lignines et les fibres mordancées au chrome ayant les TRa les plus élevés.

Mots clés : Flux digestif, transit, lapin, constituants pariétaux, lignines. 


\section{Introduction}

Parmi les différentes matières premières pouvant constituer un apport de fibres au lapin, certaines contiennent des teneurs élevées en lignines : peaux de tomates (BATrAglini \& Constantini, 1978), marc de raisin (Parigi-Bini \& Chiericato, 1980) ou pellicules de colza (LeBAs et al., 1981). Les lignines, plus ou moins fortement liées aux autres constituants pariétaux, sont habituellement considérées comme indigestibles. Elles constituent une barrière physique à l'attaque microbienne de la paroi végétale, et entraînent, chez le ruminant, une baisse de l'efficacité digestive (Jung \& FAHEY, 1983a, b).

Par ailleurs, nous ne connaissons pas, chez le lapin, la part de la digestion se déroulant dans les parties antérieures (estomac, intestin grêle) et postérieures (caecumcôlon) du tube digestif.

Nous nous proposons donc d'étudier chez le lapin, selon une technique décrite précédemment (Gidenne \& PonceT, 1985), l'effet de teneurs élevées en lignines alimentaires (8-12-16 p. 100) de rations riches en constituants pariétaux sur la digestion dans les principaux segments du tube digestif.

\section{Matériel et méthodes}

\section{A. Matériel animal et alimentation}

Au total, 108 lapereaux des deux sexes, de race Néozélandaise Blanche, répartis en trois lots égaux, sont élevés à partir de l'âge de 6 semaines en cages individuelles, dans un local éclairé $12 \mathrm{~h} / 24 \mathrm{~h}(7 \mathrm{~h} 30-19 \mathrm{~h} 30)$. Chaque lot reçoit à volonté, pendant 20 jours, l'un des trois aliments expérimentaux à base de luzerne déshydratée, remplacée en proportions croissantes par des pellicules de colza (tabl. 1). Ces trois aliments granulés, très riches en constituants pariétaux (50\% NDF), diffèrent essentiellement par leur teneur en lignines, élevée dans les trois cas : 8,0\% (régime $\mathrm{B}$ ) $; 12,0 \%$ (régime $\mathrm{M}$ ) ; $16,0 \%$ (régime $\mathrm{H}$ ). Le marqueur de transit utilisé, spécifique de la fraction pariétale de l'aliment, est l'oxyde de chrome $\left(\mathrm{Cr}_{2} \mathrm{O}_{3}\right)$ fixé par mordançage sur du foin de luzerne selon la technique décrite par UDEN (1978). Chaque aliment contient ainsi 2 p. 100 de ce foin rendu indigestible par le mordançage au chrome.

\section{B. Conduite de l'expérience et prélèvements}

Après une semaine d'accoutumance aux rations, la digestibilité apparente totale des différents composants de la ration est déterminée par collecte totale journalière des fèces durant deux périodes de 4 jours, selon la méthode de Colin \& Lebas (1976). Les fèces sont conservées en sachets de nylon étanche à $-18^{\circ} \mathrm{C}$, jusqu'à l'analyse.

Les conditions de prélèvement et la composition des contenus digestifs de ces trois lots d'animaux ont été décrites antérieurement (GIDENNE, 1987). Nous rappellerons que les digesta sont prélevés par abattage de lapins âgés de 9 semaines, à 4 niveaux du tube digestif : estomac, iléon ( 60 derniers $\mathrm{cm}$ ), caecum, rectum (30 derniers $\mathrm{cm}$ ). A partir de 9 collectes réparties toutes les 3 heures sur 24 heures, un échantillon moyen est constitué pour chaque lot, à chaque site digestif. 


\section{TABLEAU 1}

Composition des régimes expérimentaux.

Composition of the experimental diets.

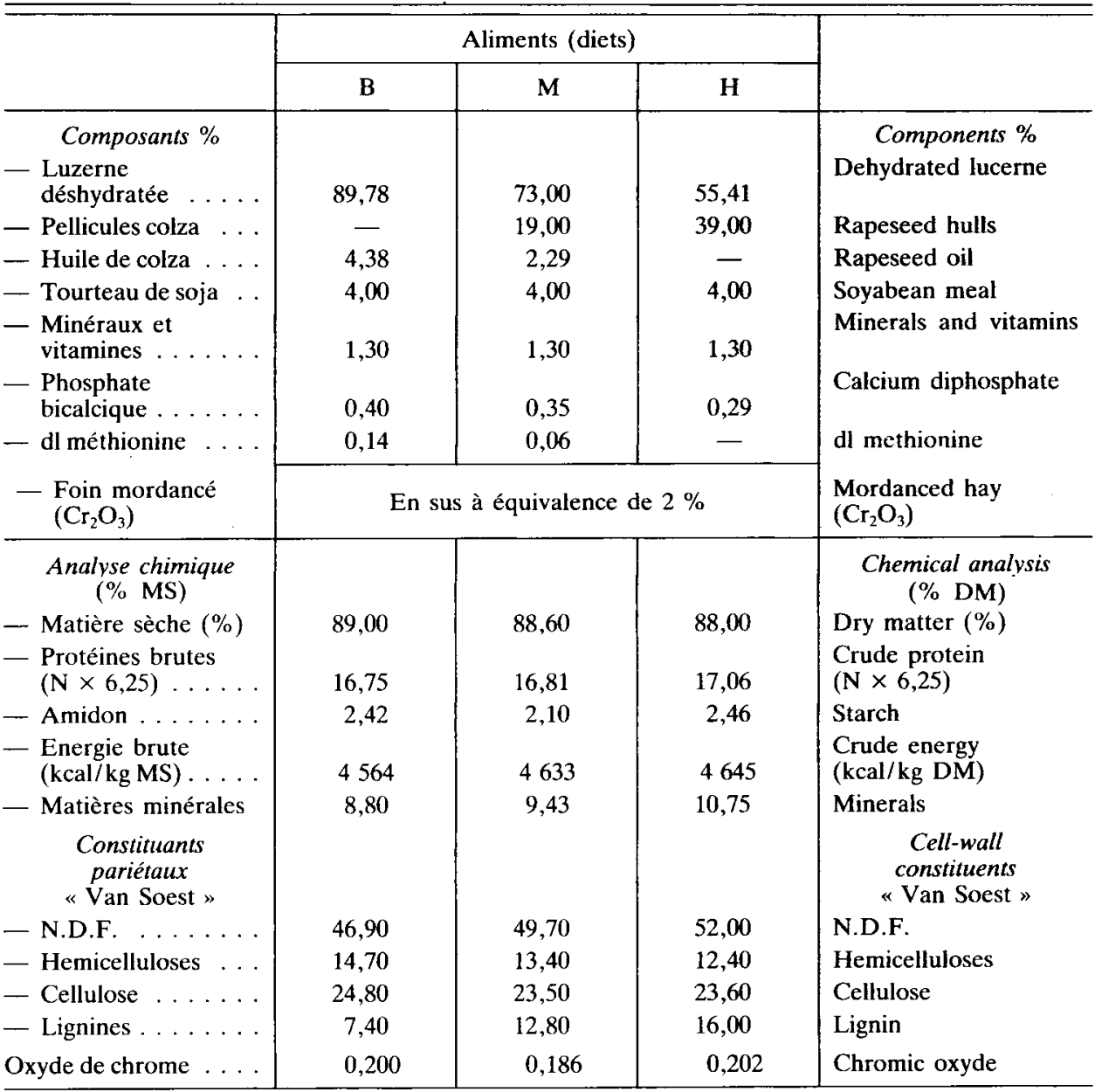

VS : Dosages effectués selon la méthode de VAN Soest \& Wine (1967).

According to the method of VAN SOEST \& WINE (1967).

\section{Analyses}

Les analyses sont effectuées sur les produits lyophilisés. La teneur en oxyde de chrome $\left(\mathrm{Cr}_{2} \mathrm{O}_{3}\right)$ a été déterminée sur les aliments, les fèces et les digesta, selon la méthode de Mathieson (1970), après minéralisation à l'acide nitrique, et oxydation par l'acide perchlorique à chaud. Nous avons également déterminé sur ces produits la teneur en matière sèche (MS) par dessication durant 24 heures à $103{ }^{\circ} \mathrm{C}$, les teneurs en 
énergie (calorimètre adiabatique PARR), et en matières azotées totales (MAT) selon la méthode de KJELDHAL $(\mathrm{N} \times 6,25)$. Le dosage des constituants pariétaux est réalisé semiautomatiquement selon la méthode de VAN SOEST \& WINE (1967), modifiée par Giger et al. (1979). Le terme "lignines" employé dans le texte s'applique donc au résidu final (corrigé pour les matières minérales) obtenu après actions successives du détergent neutre (NDS), du détergent acide (ADS) puis de l'acide sulfurique concentré $\left(\mathrm{H}_{2} \mathrm{SO}_{4}\right.$, 72 p. 100).

\section{Calcul des flux digestifs et du temps de rétention apparent (TRa)}

Le flux de MS à l'entrée ou à la sortie d'un segment digestif se calcule par la technique classique de dilution d'un marqueur indigestible.

$\mathrm{Q}(\mathrm{MS})=100 \times \mathrm{Q} / \mathrm{C}$; où $\mathrm{Q}$ représente la quantité de marqueur ayant transité en 24 h (équivalente à la quantité ingérée en $24 \mathrm{~h}$ si le taux de récupération du marqueur est de $100 \mathrm{p} .100$ ) ; et où $\mathrm{C}$ représente la concentration moyenne au cours de $24 \mathrm{~h}$ de ce marqueur dans l'organe considéré (exprimé en p. 100).

Le flux digestif $(\mathrm{g} / 24 \mathrm{~h})$ des autres composants de la ration est obtenu en multipliant la valeur du flux de MS (Q MS) par les concentrations moyennes (sur $24 \mathrm{~h}$ ) de chacun de ces composants.

A partir des valeurs des flux digestifs, la digestibilité apparente (CUDa) a été calculée pour chaque segment digestif. Les valeurs de ces CUDa partiels, ainsi que celles des CUDa totaux (collecte fécale) sont corrigées en fonction des 2 p. 100 de foin de luzerne mordancé au chrome totalement indigestible (TEETER et al., 1984).

Le temps de rétention apparent (TRa) dans l'estomac et le caecum est calculé selon la méthode de Minson (1966) :

TRa = quantité de constituant présent dans le contenu de l'organe/quantité entrant chaque heure dans l'organe digestif.

Pour ce calcul, et celui des flux digestifs, il est nécessaire d'estimer la quantité moyenne de caecotrophes ingérés chaque jour (selon Gidenne \& PonceT, 1985) car, chez le lapin, le flux réel à l'entrée de l'estomac (ingéré total quotidien) est égal à l'ingéré d'aliment, augmenté de l'ingéré de caecotrophes. Ce dernier a été calculé pour les trois lots d'animaux (Gidenne, 1987) par la méthode citée précédemment.

\section{Résultats}

A l'analyse, les trois aliments diffèrent effectivement, surtout par leur teneur en lignines $(7,4-12,8-16,0$ p. 100), conformément à celles prévues en théorie (8-12-16 p. 100 ). L'augmentation de teneur en « parois totales " (NDF) provient de l'augmentation de teneur en lignines, alors que les teneurs en hémicelluloses et cellulose sont identiques pour les trois rations.

\section{A. Ingéré, croissance et digestibilité apparente «totale » des aliments}

L'ingéré croît significativement lorsque s'élève la teneur en lignines (tabl. 2). Par contre, la détérioration de la croissance (GMQ) et de l'indice de consommation (IC) ne s'aggrave pas pour un taux de lignines supérieur à $12 \%$. 


\section{TABleAu 2}

Effet de la teneur en lignines alimentaires sur la quantité ingérée et la croissance des lapins (1). Effect of dietary lignin content on feed intake and daily weight gain of rabbits ${ }^{(1)}$.

\begin{tabular}{|c|c|c|c|c|c|}
\hline Lots & $\begin{array}{c}B \\
(n=36)\end{array}$ & $\begin{array}{c}M \\
(n=36)\end{array}$ & $\begin{array}{c}\mathrm{H} \\
(\mathrm{n}=36)\end{array}$ & \multicolumn{2}{|c|}{$\begin{array}{l}\text { Signification } \\
\text { statistique } \\
\text { (F calculé) }\end{array}$} \\
\hline $\begin{array}{l}\text { Poids en début d'expérience } \\
\text { (g) } \ldots \ldots \ldots \ldots \ldots \\
\text { Initial weight (g) }\end{array}$ & $\begin{array}{r}969 \\
+132 \\
\end{array}$ & $\begin{array}{r}987 \\
\pm 124 \\
\end{array}$ & $\begin{array}{r}960 \\
+129 \\
\end{array}$ & 0,8 & NS \\
\hline $\begin{array}{l}\text { Consommation }(\mathrm{g} / \mathrm{j}) \\
\text { Intake }(\mathrm{g} / \mathrm{d})\end{array}$ & $\begin{array}{r}125 a \\
\pm \quad 11\end{array}$ & $\begin{array}{r}129 b \\
\pm \quad 10\end{array}$ & $\begin{array}{r}136 c \\
\pm \quad 9\end{array}$ & 13,1 & $* *$ \\
\hline $\begin{array}{l}\text { Gain de poids }(g / \mathrm{j}) \ldots \\
\text { Weight gain }(g / d)\end{array}$ & $\begin{array}{r}38,9 \text { a } \\
\pm \quad 4,2\end{array}$ & $\begin{array}{r}36,2 \mathrm{~b} \\
\pm \quad 3,9\end{array}$ & $\begin{array}{r}37,3 \text { ab } \\
+\quad 2,7\end{array}$ & 5,0 & $* *$ \\
\hline $\begin{array}{l}\text { Indice de consommation } \\
\text { Feed conversion ratio }\end{array}$ & $\begin{array}{ll} & 3,46 \mathrm{a} \\
+ & 0,35\end{array}$ & $\begin{array}{ll} & 3,86 \mathrm{~b} \\
+ & 0,35\end{array}$ & $\begin{array}{r}3,93 \text { a } \\
\pm \quad 0,34\end{array}$ & 20,8 & $* *$ \\
\hline
\end{tabular}

(1) Mesures effectuées de la $6^{*}$ à la $9^{\mathbf{e}}$ semainc d'âge (21 jours).

Measurements made on rabbits from $6^{\text {th }}$ to $9^{\text {th }}$ week of age (21 days).

\section{B. Digestibilité apparente «totale» des aliments}

Comme pour le GMQ et l'IC, la digestibilité dans l'ensemble du tube digestif (CUDa calculé par récolte fécale totale) n'évolue plus au-delà d'une teneur en lignines dans l'aliment de $12 \%$ (tabl. 3). Globalement, quand la teneur en lignines de l'aliment passe de 7,4 à 12,8 p. 100 , la digestibilité apparente des constituants de la ration diminue, sauf pour l'énergie et la cellulose, dont l'utilisation digestive n'évolue pas significativement entre les trois lots. L'augmentation de la teneur en lignines de la ration diminue la digestibilité de ce constituant $(-30 \mathrm{p}$. $100 \mathrm{du}$ lot $\mathrm{B}$ à $\mathrm{M})$, qui reste toujours largement supérieure à zéro (14 à 23 p. 100). La digestibilité des matières azotées totales (MAT), déjà faible pour les lapins du lot B $(66,1$ p. 100) diminue régulièrement lorsque le taux de lignines augmente.

En supposant que la digestibilité des constituants de la ration B n'est pas modifiée par la substitution des pellicules de colza dans les rations $M$ et $H$, nous pouvons calculer la digestibilité des pellicules ; leurs protéines sont peu digestibles $(48,4 \%)$, quel que soit le taux d'incorporation de cet aliment dans la ration (18,6 et $38,1 \%)$. Leur teneur en énergie digestible varie peu : $2846 \mathrm{kcal} / \mathrm{kg}$ MS pour la ration M à 2776 $\mathrm{kcal} / \mathrm{kg}$ MS pour la ration $\mathrm{H}$.

Les digestibilités calculées à partir de collectes totales des fèces ne diffèrent pas significativement de celles obtenues à l'aide de la concentration en marqueür des fèces, quel que soit le constituant de la ration (tabl. 3).

\section{Flux digestifs et digestibilité apparente partielle}

Parallèlement à la hausse de la quantité d'aliment ingéré (tabl. 2), nous constatons une hausse des valeurs des flux, du lot $\mathrm{B}$ au lot $\mathrm{H}$ (tabl. 4). Du fait de la digestion, les 


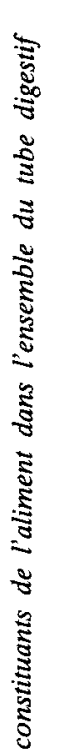

)

宅

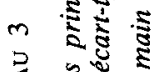

\begin{tabular}{|c|c|c|c|c|c|c|c|c|}
\hline 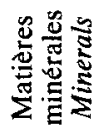 & $\begin{array}{l}\pi \\
\sigma^{2} \\
\sigma^{2}\end{array}$ & $\begin{array}{l}\pi \\
\tilde{r}_{+1}^{\infty}\end{array}$ & 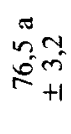 & $\begin{array}{l}\infty \\
\infty \\
\infty \\
2\end{array}$ & $\begin{array}{l}0 \\
\overrightarrow{6} \\
\hat{0}^{\prime}\end{array}$ & & & \\
\hline 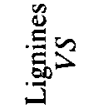 & $\begin{array}{l}n \\
n^{\infty} \\
\tilde{v}_{+1}^{\infty}\end{array}$ & $\begin{array}{l}2 \\
\hat{i}+ \\
0\end{array}$ & $\begin{array}{l}0 \\
m \\
0 \\
0 \\
0+1\end{array}$ & $\begin{array}{l}m \\
=12\end{array}$ & $\begin{array}{l}\overrightarrow{0} \\
\overrightarrow{ \pm}\end{array}$ & 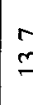 & & Z \\
\hline
\end{tabular}

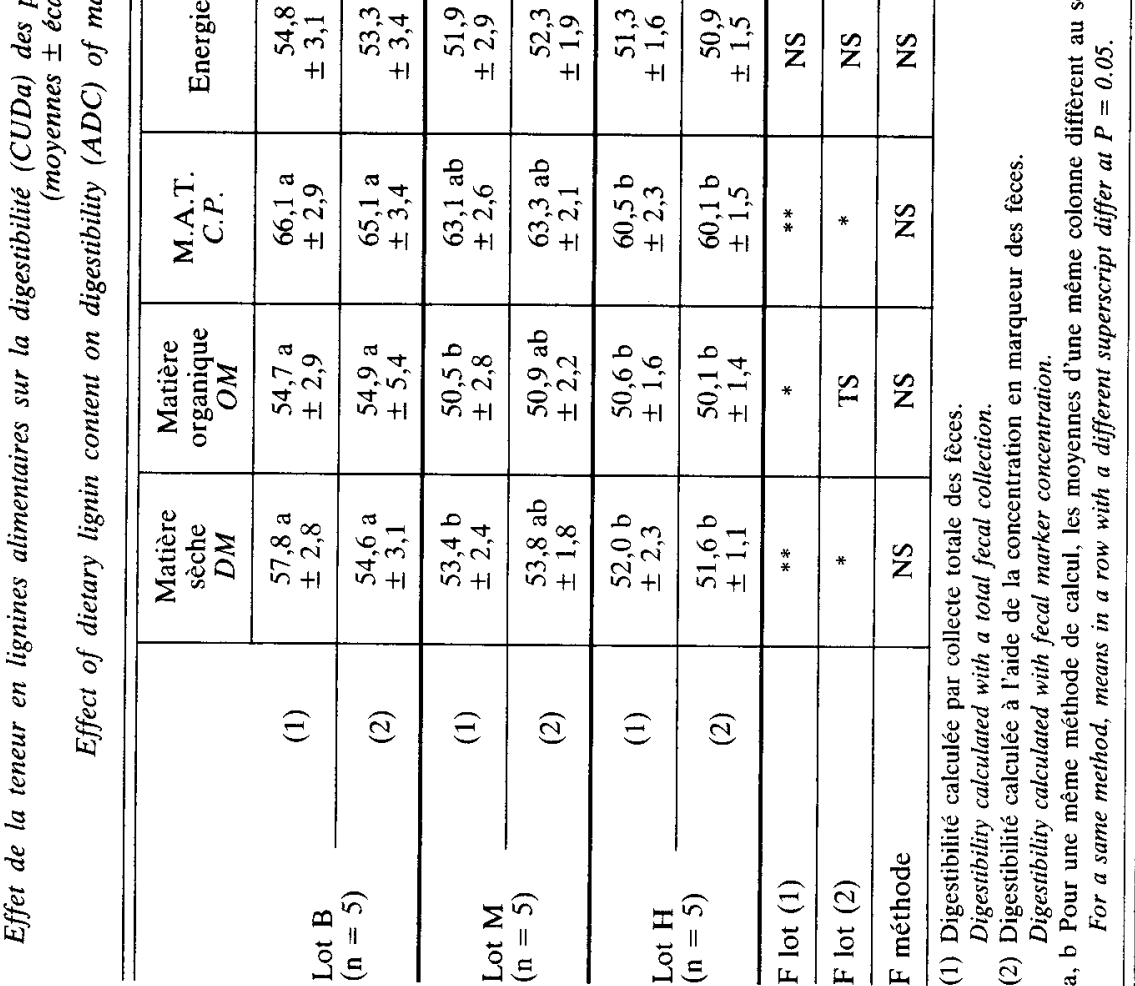




\section{TABLEAU 4}

Influence de la teneur en lignines alimentaires sur l'ingéré total $(\mathrm{g} / \mathrm{j})$ et les flux digestifs $(\mathrm{g} / \mathrm{j})$ dans différents segments du tube digestif.

Effect of dietary lignin content on total intake $(\mathrm{g} / \mathrm{d})$ and digesta flow $(\mathrm{g} / \mathrm{d})$ in different parts of digestive tract.

\begin{tabular}{l|l|c|c|c|c|c|c}
\hline \hline & & $\begin{array}{c}\text { Matière } \\
\text { sèche } \\
\text { Dry } \\
\text { matter }\end{array}$ & $\begin{array}{c}\text { M.A.T } \\
\text { Crude } \\
\text { protein }\end{array}$ & N.D.F. & $\begin{array}{c}\text { Hémi- } \\
\text { celluloses } \\
\text { N.D.F. } \\
\text { A.D.F. }\end{array}$ & $\begin{array}{c}\text { Cellulose } \\
\text { A.D.F. } \\
\text { A.D.L. }\end{array}$ & $\begin{array}{c}\text { Lignines } \\
\text { Lignin }\end{array}$ \\
\hline \multirow{2}{*}{ (1) } & Ingéré total & & & & & & \\
& Total intake & 152,4 & 28,8 & 70,7 & 21,9 & 37,5 & 11,8 \\
& Estomac/Stomach & 124,7 & 26,1 & 62,5 & 16,7 & 32,3 & 14,1 \\
& Iléon/Ileum & 145,6 & 38,2 & 55,0 & 14,0 & 30,7 & 9,9 \\
& Caecum/Cecum & 104,9 & 30,3 & 47,9 & 13,4 & 25,1 & 9,4 \\
& Rectum/Rectum & 84,1 & 15,2 & 50,1 & 13,4 & 26,2 & 10,4 \\
\hline \multirow{2}{*}{ LOT } & Ingéré total & & & & & & \\
& Total intake & 158,4 & 30,1 & 77,7 & 20,6 & 37,6 & 21,0 \\
& Estomac/Stomach & 128,9 & 24,2 & 74,4 & 16,5 & 35,8 & 21,5 \\
& Iléon/Ileum & 154,4 & 38,6 & 64,9 & 13,3 & 33,7 & 19,1 \\
& Caecum/Cecum & 110,4 & 29,2 & 56,5 & 12,9 & 27,1 & 16,8 \\
& Rectum/Rectum & 91,4 & 17,0 & 58,1 & 12,9 & 27,6 & 18,0 \\
\hline \multirow{2}{*}{$(1)$} & Ingéré total & & & & & & \\
& Total intake & 168,1 & 31,8 & 87,3 & 20,3 & 38,7 & 28,2 \\
LOT & Estomac/Stomach & 132,1 & 23,8 & 84,3 & 18,0 & 36,2 & 30,1 \\
& Iléon/Ileum & 159,5 & 35,9 & 76,6 & 13,4 & 34,0 & 28,5 \\
H & Caecum/Cecum & 124,5 & 32,2 & 64,4 & 13,7 & 26,0 & 24,6 \\
& Rectum/Rectum & 101,6 & 18,3 & 64,9 & 13,2 & 26,8 & 27,1 \\
\hline
\end{tabular}

(1) Ingéré total = Ingéré d'aliment (tabl. 2) et de caecotrophes (GidenNe, 1987),

Total intake $=$ Food intake (table 2$)$ and caecotrophes.

valeurs des flux (MS, MAT, ...) devraient diminuer des segments antérieurs aux segments postérieurs du tube digestif. Or, pour les constituants pariétaux, les valeurs calculées selon notre méthode ne vérifient pas toujours ce principe : elles baissent effectivement jusqu'au caecum, mais les valeurs mesurées dans le rectum sont supérieures ou égales à celles mesurées dans le caecum (tabl. 4). Nous avons également cherché à savoir si notre technique pouvait donner une estimation du flux de constituants plus digestibles tels que les protéines. La réponse paraît négative car, par exemple, le flux de MAT transitant dans l'iléon est supérieur à la quantité de MAT ingérée (tabl. 4).

Ces problèmes d'estimation des flux digestifs se traduisent par des digestibilités partielles aberrantes (tabl. 5). Ainsi, la digestibilité apparente de l'azote est négative dans l'intestin grêle, et celle des hémicelluloses dépasse $40 \%$ entre la bouche et l'iléon. De plus, dès l'estomac, nous observons une digestibilité relativement élevée, en particulier dans le cas des hémicelluloses. Cependant, les digestibilités calculées à partir des digesta prélevés dans le rectum (tabl. 5) sont équivalentes à celles obtenues par collecte totale des fèces (tabl. 3). 
Etant donné la cohérence des résultats entre les trois lots, il est possible de les comparer pour évaluer l'effet de la ration. Ainsi, la hausse de la teneur en lignines de l'aliment semble augmenter la digestion caecale (flux iléal-flux caecal/ingéré) des constituants pariétaux. Dans le cas de la cellulose, elle passerait de 18,5 à $24,5 \%$ du lot $\mathrm{B}$ au lot $\mathrm{H}$. Pour les lignines, ces proportions seraient de 5,4 et $18,3 \%$.

\section{TABLEAU 5}

Effet de la teneur en lignines alimentaires sur la digestibilité apparente (\%) dans différents segments du tube.

Effect of dietary lignin content on apparent digestibility in different parts of digestive tract.

\begin{tabular}{l|l|c|c|c|c|c|c}
\hline \hline & & $\begin{array}{c}\text { Matière } \\
\text { sèche } \\
\text { Dry } \\
\text { matter }\end{array}$ & $\begin{array}{c}\text { M.A.T } \\
\text { C.P. }\end{array}$ & N.D.F. & $\begin{array}{c}\text { Hémi- } \\
\text { celluloses } \\
\text { N.D.F.- } \\
\text { A.D.F. }\end{array}$ & $\begin{array}{c}\text { Cellulose } \\
\text { A.D.F.- } \\
\text { A.D.L. }\end{array}$ & $\begin{array}{c}\text { Lignines } \\
\text { Lignin }\end{array}$ \\
\hline \multirow{4}{*}{ LOT } & Estomac/Stomach & 22,7 & 13,0 & 14,2 & 29,2 & 17,2 & $-25,9$ \\
B & Iléon/lleum & 5,6 & $-46,0$ & 27,3 & 44,4 & 22,5 & 20,6 \\
& Caecum/Cecum & 38,9 & $-7,3$ & 39,7 & 47,5 & 41,0 & 26,2 \\
& Rectum/Rectum & 56,0 & 66,3 & 35,8 & 47,6 & 37,4 & 14,8 \\
\hline \multirow{4}{*}{ LOT } & Estomac/Stomach & 23,3 & 27,7 & 5,8 & 24,3 & 6,0 & $-2,8$ \\
M & Iléon/Ileum & 3,2 & $-40,0$ & 20,3 & 43,3 & 13,2 & 12,0 \\
& Caecum/Cecum & 38,0 & 4,2 & 33,2 & 45,5 & 35,1 & 26,2 \\
& Rectum/Rectum & 53,0 & 61,6 & 31,3 & 45,6 & 33,6 & 18,8 \\
\hline \multirow{4}{*}{ LOT } & Estomac/Stomach & 27,0 & 35,8 & 4,3 & 14,5 & 8,0 & $-8,9$ \\
H & Iléon/lleum & 6,4 & $-17,8$ & 15,4 & 42,1 & 15,1 & $-1,6$ \\
& Caecum/Cecum & 32,7 & $-1,6$ & 33,0 & 40,2 & 40,5 & 16,7 \\
& Rectum/Rectum & 49,9 & 59,4 & 32,3 & 43,3 & 37,8 & 5,2 \\
\hline \hline
\end{tabular}

\section{Temps de rétention apparents (TRa) dans l'estomac et le caecum}

Outre les TRa des constituants de la paroi, nous présentons le TRa calculé pour les fibres mordancées qui correspondent à la fraction indigestible de la ration. Etant donné les problèmes rencontrés pour l'estimation des flux iléaux de MS et de MAT, nous n'avons pas calculé le TRa de ces constituants dans le caecum. Dans le cas des constituants pariétaux, nous avons effectué ce calcul en supposant que la digestion des constituants pariétaux serait négligeable avant le caecum; en conséquence, le flux entrant dans le caecum est équivalent à l'ingéré total «aliment et caecotrophes ».

Le TRa mesuré dans le caecum est supérieur d'environ $1 \mathrm{~h}$ à $2 \mathrm{~h}$ à celui mesuré dans l'estomac, excepté dans le cas de la ration la plus riche en lignines (lot $\mathrm{H}$ ) (tabl. 6). Que ce soit dans l'estomac ou le caecum, ce sont les fractions les plus indigestibles de l'aliment (fibres mordancées au chrome, lignines) qui présentent les temps de rétention les plus longs. 
TABLEAU 6

Effets de la teneur en lignines sur le temps de rétention apparent (heures) des constituants alimentaires dans l'estomac et le caecum.

Effects of lignin content on apparent retention time (hours) of dietary components in stomach and cecum.

\begin{tabular}{l|l|c|c|c|c|c|c|c}
\hline \hline & & $\begin{array}{c}\text { Matière } \\
\text { sèche } \\
\text { Dry } \\
\text { matter }\end{array}$ & $\begin{array}{c}\text { M.A.T } \\
\text { C.P. }\end{array}$ & N.D.F. & $\begin{array}{c}\text { Hémi- } \\
\text { cellu- } \\
\text { loses } \\
\text { N.D.F.- } \\
\text { A.D.F. }\end{array}$ & $\begin{array}{c}\text { Cellu- } \\
\text { lose } \\
\text { A.D.F.- } \\
\text { A.D.L. }\end{array}$ & Lignines & $\mathrm{Cr}_{2} \mathrm{O}_{3}$ \\
\hline \multirow{3}{*}{$\begin{array}{l}\text { Estomac } \\
\text { Stomach }\end{array}$} & Lot B & 1,7 & 1,9 & 1,9 & 1,7 & 1,8 & 2,5 & 2,1 \\
\cline { 2 - 10 } & Lot M & 2,7 & 2,7 & 2,7 & 2,7 & 3,2 & 3,5 & 3,3 \\
\cline { 2 - 10 } Caecum & Lot $\mathrm{H}$ & 2,9 & 2,8 & 3,6 & 3,3 & 3,5 & 4,0 & 3,6 \\
\cline { 2 - 9 } Cecum & Lot B & - & - & 3,5 & 3,2 & 3,5 & 4,2 & 5,0 \\
\cline { 2 - 9 } & Lot M & - & - & 3,7 & 3,1 & 3,6 & 4,0 & 5,1 \\
\hline
\end{tabular}

Avec l'accroissement du taux de lignines, le TRa dans l'estomac augmente de +50 à +94 p. 100, selon le constituant. Dans le caecum, les écarts sont beaucoup plus faibles ( 5 à $25 \%$ ), et le phénomène précédent est inversé : baisse du TRa quand le taux de lignines augmente. Ainsi, dans le cas du lot B, les TRa dans l'estomac sont équivalents à environ la moitié de ceux observés dans le caecum. Par contre, dans le cas du lot $\mathrm{H}$, les TRa de l'estomac et du caecum sont beaucoup plus proches, voire identiques.

\section{Discussion}

\section{A. Digestibilité des rations et croissance des animaux}

Bien que la teneur en lignines des aliments varie de 7,4 à $16 \%$, la croissance des lapins n'est pas fortement modifiée. En effet, les animaux semblent ajuster leur consommation à leur besoin : la quantité d'énergie digestible (ED) et de protéines digestibles (PD) ingérée ne diffère pas significativement entre lots $(310 \mathrm{kcal} \mathrm{ED} / \mathrm{j}$ et $13,6 \mathrm{PD} / \mathrm{j}$, en moyenne). Cette régulation de la consommation d'ED avait déjà été observée par Lebas (1975) et par SPREAdBuRy \& Davidson (1978) sur des lapins nourris avec des régimes à teneurs en cellulose brute variables, mais dont la teneur en lignines ne dépassait pas $5 \%$.

Il est possible d'estimer le CUDa d'un nutriment, simplement à l'aide de la teneur en marqueur d'un échantillon de fèces. Ceci évite une récolte totale de ces dernières. Toutefois, les écarts de digestibilité qu'il est possible de mettre en évidence avec la technique de récolte totale sont plus faibles (écart-type inter-individuel réduit). 
Les teneurs élevées en constituants pariétaux et en lignines des rations expliquent vraisemblablement la faible digestibilité de la MS, de la MO et de l'énergie, comme l'ont déjà montré Lebas (1975), Colin \& Allain (1978) et Lebas et al. (1982). Mais, d'après ces mêmes auteurs, la teneur en fibres (méthode de WEENDE) du régime n'affecte pas la digestibilité apparente des protéines. Par contre, cette dernière diminue quand la teneur en lignines du régime augmente, comme l'indiquent nos résultats, et en accord avec ceux de Parigi-Bini \& Chiericato (1980), Battaglini \& Constantini (1978), Lebas et al. (1981), Falcao E Cunha \& Lebas (1986).

Cet effet des lignines peut avoir diverses origines: inhibition de l'activité des enzymes digestives, formation de polymères indigestibles avec les protéines, ou encore inhibition des fermentations caecales. Pour ce dernier point, nous avons déjà montré (GiDENNE, 1986) que les lignines de nos rations ne semblaient pas jouer un rôle inhibiteur majeur. Quant à la formation de polymères indigestibles, nous pouvons dire que les pellicules de colza apportent 47 et $73 \%$ des lignines respectivement dans les aliments $\mathrm{M}$ et $\mathrm{H}$, et que le résidu lignines extrait à l'acide sulfurique contient, d'après THEANDER et al. (1977) une importante proportion de tannins condensés pouvant former des complexes indigestibles avec les protéines (Mc LeOD, 1974). CARre \& BRILlouet (1986) montrent que les $2 / 3$ des MAT des parois d'un tourteau de colza non dépelliculé sont associés aux lignines, soit environ $15 \%$ de l'azote des pellicules de colza. Le cumul de ces deux phénomènes est sans doute à l'origine de la faible digestibilité des protéines que nous avons calculée pour les pellicules de colza.

Contrairement à nos résultats, Falcao E Cunha \& Lebas (1986) indiquent que le CUDa des lignines provenant des peaux de tomates et de marc de raisin, s'élève avec leur teneur dans l'aliment. Pour Crampton \& Maynard (1938) et Furrer (1966), les lignines apparaissent pratiquement indigestibles chez le lapin. Lenz \& Schurch (1967) montrent que, contrairement à la lignine native, la lignine isolée du bois peut être « digestible » à $40 \%$. L'origine des lignines influence donc fortement leur propre digestibilité apparente. De plus, la méthode de dosage des lignines est un important facteur de variation des résultats obtenus (Giger, 1985). Ainsi, dans le cas des rations $\mathbf{M}$ et $\mathbf{H}$, le résidu «lignines » que nous isolons par la méthode de VaN Soest, devrait plutôt être considéré comme un résidu " lignines + tannins ". Ceci est sans doute une des raisons pour lesquelles nous obtenons une digestibilité non nulle des lignines. En outre se pose le problème de l'emploi d'une même technique (telle que celle de VAN SoEst, mise au point pour les fourrages) dans le cas de substrats différents : aliments, fèces, digesta,... Il faut donc savoir si la digestibilité des lignines correspond à une véritable absorption ou à la non-détection de produits transformés au cours du transit digestif.

D'autre part, la digestibilité des autres constituants pariétaux (cellulose-hémicelluloses) est variable selon le nombre et le type de liaisons établies avec les lignines. Ainsi, les hémicelluloses contenues dans nos trois rations sont plus digestibles que la cellulose, comme le constatent Uden \& VAN Soest (1982) et Randall (1977). Par contre, Falcao E Cunha \& Lebas (1986) obtiennent des résultats inverses. De plus, selon ces auteurs, une augmentation de la teneur en lignines (composées en partie de tannins) dans l'aliment est associé à un accroissement significatif de la digestibilité apparente des hémicelluloses et de la cellulose. 


\section{B. Flux digestif et transit}

Notre méthode ne fournit pas une estimation exacte des flux digestifs dans le cas de certains compartiments digestifs chez le lapin. Ainsi, elle conduit à des digestibilités élevées des constituants pariétaux, dès l'estomac, la teneur en marqueur du contenu de cet organe étant supérieure à celle de l'iléon (GIDENNE, 1987), comme l'ont déjà signalé d'autres auteurs; il en résulte une sous-estimation du flux et une digestibilité trop élevée. Comme nous l'avons décrit (Gidenne \& Poncet, 1985), ce phénomène s'explique par une rétention des caecotrophes dans l'estomac plus longue que celle de l'aliment, et par un temps de séjour gastrique de la fraction soluble de l'aliment plus court que celui de la fraction particulaire. Du fait de ce transit différentiel, une mesure de flux dans l'estomac n'est pas possible, bien que nous ayons prélevé la totalité du contenu de ce site pour éviter les erreurs d'échantillonnages (fréquents dans le cas de compartiments de mélange).

Le problème de l'hétérogénéité des digesta ne se pose pas dans le cas de l'iléon ou de caecum, dont les contenus sont homogènes (Yoshinara \& Kandatsu, 1960, cité par LAPLACE, 1978). Ainsi, notre raisonnement, valable pour l'estomac, n'explique pas que les flux mesurés dans le caecum soient équivalents ou inférieurs à ceux mesurés dans le rectum, ni la digestibilité iléale très élevée des hémicelluloses. Nos résultats portant sur les TRa indiquent que les différents constituants pariétaux n'ont pas un transit identique, au moins aux niveaux stomacal et caecal. Or, les contenus digestifs sont prélevés par abattage. Nous n'avons donc qu'une image instantanée de la composition du contenu des différents segments digestifs qui ne rendrait pas compte des éventuelles vitesses de transit différentielles des constituants de l'aliment (KAMETAKA, 1957 ; BJORNHAG, 1972). De plus, une erreur sur la composition chimique est davantage importante dans le cas de segments digestifs tubulaires tel que l'iléon où les vitesses de transit sont assez élevées et la quantité de contenu faible. D'autre part, la desquamation de l'épithélium intestinal après abattage, peut augmenter la part d'azote endogène, et donc le flux d'azote à ce niveau. Ces erreurs d'échantillonnages paraissent être la cause majeure d'une mesure inexacte de flux digestif.

L'estimation correcte des flux digestifs au niveau du rectum (CUDa partiels voisins de ceux obtenus par collecte fécale totale) confirme ce point de vue. En effet, dans ce site, le phénomène de transit différentiel des constituants est négligeable, puisqu'il s'agit de digesta sous forme de crottes (dures ou molles) : ceci montre la validité de notre principe du calcul de flux digestif chez le lapin qui nécessite une mesure de l'ingéré total «aliment et caecotrophes ». Le problème posé est donc celui de la représentativité des échantillons de contenus digestifs collectés par rapport au matériel digestif qui transite en un site donné.

LEBAS (1979) estime, d'après la répartition d'un marqueur dans le tube digestif, que le temps de séjour des aliments est plus long dans le caecum (6 à 9 h) que dans l'estomac (4 à $6 \mathrm{~h})$. Nos résultats s'accordent avec cette hiérarchie " estomac-caecum ", mais indiquent un transit plus rapide. Outre l'effet de la méthode de mesure, cet écart peut provenir de la teneur importante en fibres de nos régimes. En effet, selon LeBAS \& LAPLACE (1977), le temps de séjour diminue lorsque la teneur en cellulose brute (CB) de la ration augmente. De plus, nous estimons le TRa des constituants pariétaux, alors que les valeurs proposées par LEBAS (1979) concernent la ration dans son ensemble. Ce transit rapide, associé au faible niveau des digestibilités, est sans doute un facteur atténuant l'effet propre des lignines. Enfin, nous constatons que la baisse des CUDa des constituants pariétaux entre les lots $B$ et $\mathbf{M}$ (excepté pour la cellulose), n'est pas accompagnée d'une baisse équivalente des TRa caecaux correspondants. 


\title{
V. Conclusion
}

La teneur élevée en constituants pariétaux et en lignines de nos régimes rend compte du niveau généralement modeste des digestibilités enregistrées. Celà est associé à un temps de rétention relativement court des constituants pariétaux de la ration dans l'estomac et le caecum.

De ce fait, l'effet inhibiteur des lignines sur l'efficacité digestive des lapins est sans doute moins perceptible. Toutefois, quand la teneur en lignines de l'aliment passe de 7,4 à $12,8 \%$, les paramètres de la digestibilité de la ration sont significativement plus faibles, excepté la digestion de l'énergie et de la cellulose. Parallèlement, les TRa mesurés dans l'estomac augmentent, alors qu'ils varient peu au niveau caecal.

Notre méthode d'étude basée sur l'abattage, à différents moments de la journée, de lapins nourris avec un aliment marqué, ne paraît pas adéquate pour les mesures de flux digestifs, particulièrement dans les sites digestifs où les constituants de la ration sont susceptibles de transiter à des vitesses différentes, et dans lesquels les contenus sont quantitativement faibles.

Le problème de l'estimation des flux digestifs (en particulier dans le cas de l'iléon) semble résider surtout dans le prélèvement de digesta représentatifs du flux. La collecte des digesta à l'aide de canules serait susceptible de résoudre ce type de problème, dans la mesure où la totalité du flux (phases liquide et solide) sera récoltée pendant une période donnée, et non pas de manière instantanée, comme c'est le cas après abattage.

Reçu en février 1987.

Accepté en avril 1987.

\begin{abstract}
Summary
Apparent digestibility of high-lignin diets in the growing rabbit : transit and digesta flow measurements in different parts of the digestive tract
\end{abstract}

Breakdown of cell-wall constituents and their effect on digestion have not been well established in the rabbit. The effect of high-lignin contents on digestibility, flux and digestive transit were measured in three groups of 36 young rabbits fed high-fibre diets (50\% NDF) based on dehydrated lucerne and rapeseed hulls, mainly differing in their lignin VAN SOEST concentration : 7.4 (diet B), 12.8 (diet M) and 16\% DM (diet H). Transit and digesta flow measurements were performed using a technique combining slaughters distributed over a $24 \mathrm{~h}$ period after ingestion of a labelled feed (mordanced chromic oxyde).

Increasing lignin concentration led to a moderate decrease in digestibility : protein digestibility was more affected $(\mathrm{ADC}=66.1 \%$ with diet $\mathrm{B}$ versus $60.5 \%$ with diet $\mathrm{H})$, whereas energy and cellulose apparent digestibility were not significantly affected (table 3 ). Apparent digestibility of lignin, reputedly undigestible, varied between 14.0 (diet $\mathrm{H}$ ) and $23.0 \%$ (diet B). The application of VAN SOEST's method, particularly for analysing faeces or by-products such as rapeseed hulls is discussed.

According to ileal flow (table 4), hemicellulose digestibility was high, whereas crude protein digestibility was negative (table 5). However, digesta flow measured in the rectum was in agreement with values obtained by total faecal collection : in this site, digesta (in the form of hard and soft faeces) dit not exhibit differential transit rates. Digesta collected after slaughtering were 
not representative (except in the rectum), but the principle of flow measurement taking into account caecotrophy is confirmed.

Apparent retention time of cell-wall constituents (table 6) varied between 3 to $5 \mathrm{~h}$ in the caecum and 2 and $4 \mathrm{~h}$ in the stomach. Increasing Lignin concentration increased values for stomach, therefore differences between stomach and caecum decreased. The more undigestible (Lignin and mordanced Fibre) fraction hat the highest retention time.

Key words : Digesta flow, transit, rabbit, cell-wall constituent, lignin.

\section{Références bibliographiques}

Battaglini M., Constantini F, 1978. Residui della lavorazione industriaie del promodoro nelle diete per coniglio in accrescimento. Coniglicoltura, 15, 19-22.

BJörnhag G., 1972. Separation and delay of contents in the rabbit colon. Swedish J. agric. Res., 2, $125-136$.

Carre B., Brillouet J.M., 1986. Yield and composition of cell-wall residues isolated from various feedstuffs used for non ruminant farm animals. J. Sci. Food. Agric., 37, 341-351.

Colin M., Lebas F., 1976. Méthode d'étude de la digestibility des aliments chez le lapin. II/ Périodicité des collectes. Sci. Tech. Anim. Labo., 1, 129-133.

Colin M., Allain, 1978. Etude du besoin en lysine du lapin en croissance, en relation avec la concentration énergétique de l'aliment. Ann. Zootech., 27, 17-31.

Crampton E.W., Maynard L.A., 1938. The relation of cellulose and lignin content to the nutritive value of animal feeds. J. Nutr., 15, 383-395.

Falcao E Cunha, Lebas F., 1986. Influence chez le lapin adulte de lorigine et du taux de lignines alimentaire sur la digestibilité de la ration et l'importance de la caecotrophie. $4^{\mathrm{e}}$ Journées de la Recherche Cunicole en France, communication 8.

FüRRER O.F., 1966. Uber die Stoffliche und Energetische Wirkung einiger Kohfaserkomponenten beim kaninchen. Thèse, Université Zürich, 77 p.

GidenNE T., 1986. Evolution nycthémérale des produits de la fermentation bactérienne dans le tube digestif du lapin en croissance. Relation avec la teneur en lignines de la ration. Ann. Zootech., 35, 121-136.

GidenNE T., 1987. Influence de la teneur en lignines des aliments sur la composition des digesta et la production de caecotrophes chez le lapereau. Ann. Zootech., 36, (sous presse).

Gidenne T., Poncet C., 1985. Digestion chez le lapin en croissance, d'une ration à taux élevé de constituants pariétaux : étude méthodologique pour le calcul de digestibilité apparente par segment digestif. Ann. Zootech., 34, 429-446.

Giger S., Sauvand D., Dorleans M., Morand-Fehr P., 1979. Détermination semi-automatique des constituants membranaires des aliments concentrés par la méthode de VAN-SOEST. $3^{\text {th }}$ Annual Meeting of the European Association for animal production, Harrogate, England, 2326 July 1979.

Giger S., 1985. Revue sur les méthodes de dosage de la lignine utilisées en alimentation animale. Ann. Zootech., 34, 85-118.

JUNG H.G., FAHEY G.C., 1983a. Nutritional implications of phenolic monomers and lignin : a review. J. Anim. Sci., 57, 206-219.

JUNG H.G., FAHEY G.C., 1983b. Inhibitory effect of phenolic monomers on fermentation of structural carbohydrates by rumen microorganisms. J. Anim. Sci, 57, suppl. no 1, 287-288.

KametaKA M., 1957. Studies on the digestion in the rabbit. $\mathrm{X} / \mathrm{On}$ the distribution of feed constituants in the contents of the alimentary tract. Jap. J. Zootech. Sci., 28, 219-224.

LAPlace J.P., 1978. Le transit digestif chez les monogastriques. III/ Comportement (prise de nourriture, caecotrophie), motricité et transit digestif et pathogénie des diarrhées chez le lapin. Ann. Zootech., 27, 225-265. 
Lebas F., 1975. Influence de la teneur en énergie de l'aliment sur les performances de croissance du lapin. Ann. Zootech., 24, 281-288.

Lebas F., 1979. La nutrition du lapin : mouvement des digesta et transit. Cuniculture, 6, 67-68.

LeBas F., LaPLACE J.P., 1977. Growth and digestive transit in the rabbit. Variations determined by physical form, composition and crude fiber content of the feed. Ann. Biol. anim. Biochim. Biophys., 171, 535-538.

Lebas F., Seroux M., Franck Y., 1981. Utilisation de pellicules de colza dans l'alimentation du lapin en croissance. I/ Performance d'engraissement. Ann. Zootech., 30, 313-323.

Lebas F., Laplace J.P., Droumene P., 1982. Effets de la teneur en énergie de l'aliment chez le lapin. Variations en fonction de l'âge des animaux et de la séquence des régimes alimentaires. Ann. Zootech., 31, 233-256.

LeNZ F.J., Schurch A., 1967. Uber das Verhalten von lignin im verdauungstrakt des Kaninchens. Z. Tierphysiol. Tierernähr. Futtermittelk, 22, 236-241.

Mathieson J., 1970. Automated estimation of chromicoxyde. Proc. Nutr. Soc., 29, 30a.

Mc Leod M.N., 1974. Plant tannins. Their role in forage quality. Nutr. Abst. Rev., 44, 803-815.

Minson D.J., 1966. The apparent retention of food in the reticulorumen at two levels of feeding by means of an hourly feeding technique. Br. J. Nutr., 20, 767-773.

Parigi-Bini R., Chiericato F., 1980. Utilisation of grape marc by growing rabbits. $2^{c}$ congrès mondial de cuniculture, Barcelone, avril 1980, vol. II, 204-213, Ed., Ac. Es. Cu. Barcelone.

RaNDALl K.E., 1977. Digestion of dietary fiber and energy in the adult New-Zealand white rabbit. Thesis, Aberdeen University, Uk., 26 p.

Spreadbury D., Davidson J., 1978. A study of the need for fibre by the growing New Zealand White rabbit. J. Sci. Fd. Agric., 29, 640-648.

Teeter R.G., OWens F.N., Mader T.L., 1984. Ytterbium chloride as a marker for particulate matter in the rumen. J. Anim. Sci., 58, 465-473.

Theander O., Aman P., Milksche G.E., 1977. Carbohydrates, polyphénols and lignin in seed hulls of different colors from turnip rapeseed. J. Agric. Food. Chem., 25, 270-273.

UDEN P., 1978. Comparative studies on rate of passage, particle size and rate of digestion in ruminants, equines, rabbits and man. Thesis, Cornell University, PH. D. 165 p.

Uden P., VAN Soest P.J., 1982. Comparative digestion of timmothy (Phleum pratense) fibre by ruminants, equines and rabbits. Bri. J. Nutr., 47, 267-272.

VAN Soest P.J., WINE R.H., 1967. Use of detergents in the analysis of fibrous feeds. IV/ Determination of plant cell-wall constituent. J. Ass. Off. Agric. Chem., 50, 50-55.

Yoshinara I., Kandatsu M., 1960. Studies on cecum digestion. 4/ On the movement of caecal contents in the rabbit. Bull. Agr. Chem. Soc., Japan. 